\title{
New microscopy based on liquid crystals and its application to students' education and researches
}

\section{Tomilin}

M. G. Tomilin, "New microscopy based on liquid crystals and its application to students' education and researches," Proc. SPIE 9666, 11th Education and Training in Optics and Photonics Conference, 96661Q (5 June 2009); doi: $10.1117 / 12.2208083$

SPIE Event: Eleventh International Topical Meeting on Education and Training in Optics and Photonics, 2009, St. Asaph, United Kingdom 


\title{
New microscopy based on liquid crystals and its application to students' education and researches
}

\author{
M.G. Tomilin \\ St.-Petersburg University of Information Technologies, Mechanics and Optics, St.- \\ Petersburg, 197101, Kronverksky Pr. 49, Russia
}

\begin{abstract}
The optical microscope operation is limited with illumination distribution detecting on the object surface in reflective regime and absorption object parameters detecting in transparent regime. The functions are increased by observation the objects in polarizing light. The inside tension and optical activity in transparent materials becomes visible. Optical polarizing microscopy is powerful tool for investigations in many fields of science and technology. But it is helpless in detecting invisible physical fields' distribution on the object surface. The combination of optical polarizing microscope with liquid crystal spatial light modulator in contact with objects' increases its functions.

The novelty of microscope consists in LC layer introduction in optical scheme to observe its local deformations in real time. LC applied as recording media has to be in contact with the surface under investigation. In this case LC detects the invisible physical fields on the object's surface: intermolecular interactions, electrical, magnetic fields, etc. The results were obtained with high optical resolution and sensitivity.

The operation with new microscope is very simple. The unique information was received in examination the surfaces of solid crystals, minerals, metals, semiconductors, polymers, glasses, optical coatings. The most valuable information was obtained in biophotonics.

The simplicity of new microscopic methods made possible to recruit for serious scientific investigations the students from first to fifth year of education. Students' participation helps to get rich statistic results and to check their reproducibility. The students also got experience in oral presentations of the results.
\end{abstract}

Keywords: polarizing microscope, liquid crystals, visualization, invisible physical fields, students' experiments, seminars and oral presentations, scientific publications.

\section{INTRODUCTION}

The education becomes most effective when the teacher excite the deep interest of pupils to the subject of investigation. The achievement of such result becomes possible if the learner takes place in real scientific problem creative search. The collaboration of supervisor with students gives fruitful results for both sides. Students' participation helps to get rich statistic results and to check their reproducibility. The students got experience in oral presentations of the results. They also receive practical skills in righting abstracts and paper under teacher's guidance. The active scientific studies at early ages usually give the main direction for whole future field of activity.

In our paper we will share with our experience on collaboration with the students from first to fifth year of education. The program for the 4-th year of education in physics and material science consist the special discipline called training students investigation that include theoretical study, experiments and oral presentations at department seminar. The seminars are organized as serious scientific action including critical opponent's remarks and common discussion of results. The results obtained during five months of training course become often the subject of diploma investigation.

In such case the choice of optical scientific problem is very important task. We decided to use for students training new optical polarizing microscope based on nematic liquid crystals (NLC) with very wide field of application. In our paper we discuss the idea of new microscope, its applications in science and high 
technologies and investigation results obtained together with the students. We consider the studies fulfilled with new optical microscope as a good example of education and training in optics and photonics.

\section{THE BASIC PRINCIPLE OF NEW OPTICAL POLARIZING MICROSCOPE BASED ON NLCS}

Optical microscope functions are limited by illumination distribution observation on the objects' surface in reflexion regime or absorption observation in transmissive regime. Polarizing microscope functions are expanded: internal tensions or optical chirality's in transparent material becomes visible. Unfortunately traditional optical microscopy doesn't give the possibility to detect the distribution of invisible physical fields such as electric, magnetic fields or structure inhomogeneities on the surface under investigation.

We suggested new type of optical polarizing microscope (OPM) with more expanded functions that gives the possibilities to observe and detect the distribution of invisible physical fields and structural defects on the materials surface. The new instrument opens new possibilities in optical metrology for detecting the quality of materials in science, technology and commercial products.

The recording of the physical fields at the surface of investigation and surface structural inhomogeneities becomes possible if the NLC deformed layer is illuminated in transmissive or reflective modes and observed through OPM and appearing figure is compared to the background structure. NLCs are more sensitive to physical fields influence in comparison with cholesteric and smectic LCs having super molecular structure.

The principle scheme of the NLC technique in combination with polarizing microscopy is shown on fig. 1.
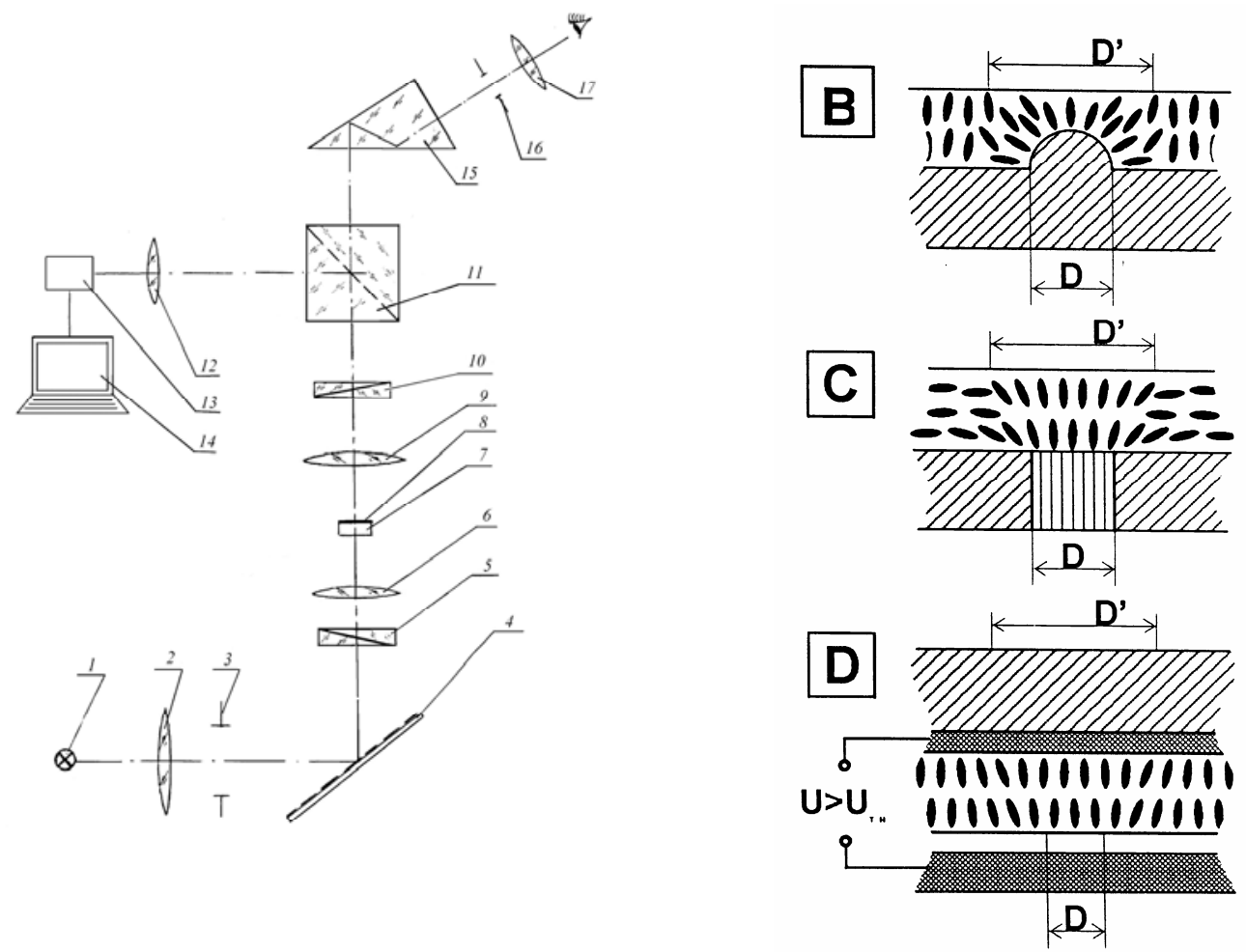

Fig.1. Optical scheme: 1-light source, 2-collector, 3-aperture, 4-mirror, 5-polarizer, 6-condenser lens, 7-object, 8-LC SLM, 9-microscope lens, 10-analyzer, 11-prism-cube, 12-ocular 1, 13-CCD-sensor, 14-computer, 15-prism, 16-aperture, 17ocular. B, C, D - defects types. D - real size of defect. D'- image size of defect in NLC.

New OPM may be also combined with interference, phase-contrast and even near-field microscopes. The light intensity over NLC layer $I(x, y)$ modulated by deformed NLC structure is described by equation:

$$
I(x, y)=I_{0} \operatorname{Sin}^{2}[\delta(x, y) / 2]
$$


The phase delay $\delta(x, y)$ caused by the NLC birefringence is equal to:

$$
\delta(x, y)=2 \pi / \lambda\left[-n_{0} \cdot H+\int_{0}^{H} n(x, y, z) d z\right]
$$

Here $H$ is the thickness of NLC film; $n(x, y)$ is the film reflective index of deformed zone; $n_{0}$ is the refractive index of a non-deformed layer. If the orientation field has no twist deformation then only orientation bending occurs, hence:

$$
n(x, y, z)=\left[n_{e}^{-2} \operatorname{Sin}^{2} \varphi(x, y, z)+n_{0}^{-2} \operatorname{Cos}^{2} \varphi(x, y, z)\right]^{1 / 2}
$$

Here $\varphi(x, y, z)$ is the deflection angle of the long axis of the molecules with the respect to the surface normal; $n_{o}, n_{e}$ are the refractive indices of NLC layer for ordinary and extraordinary polarization. The usual value of NLC optical anisotropy has the value $0,05 \ldots 0,2$ but in extreme cases it may be up to 0,4 . It permits to use very thin layers to obtain sufficient value of phase delay ${ }^{1}$.

Summarizing the principle of OPM operation we observe in polarized light not the invisible structural defect $D$, but only the deformations in LC layer D' induced by defect (fig.2, left). In non polarized light we observe the real structural defect $D$ through transparent LC layer and see nothing (fig.2, right).

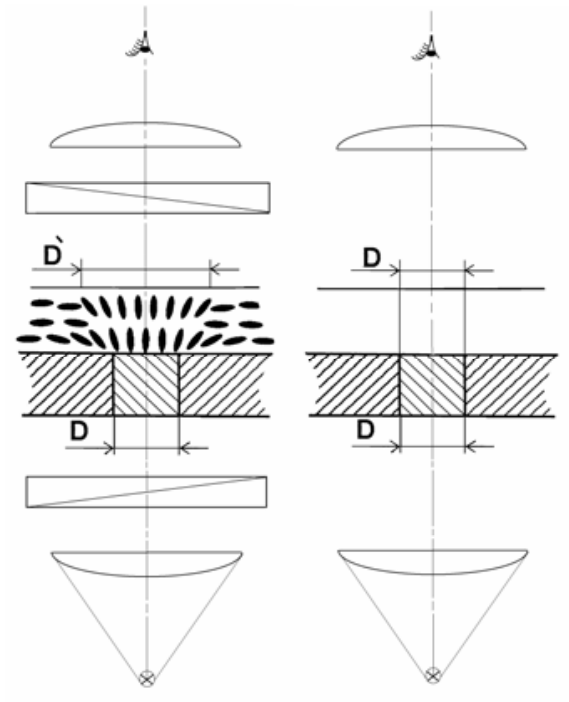

Fig.2. The principle of OPM operation.

The theory of LC layer deformations near the structural defects or obtained by magnetic or electrical fields is developed and described in papers ${ }^{2,3}$.

The most interesting and practically important case is structural inhomogeneities detecting (fig.1C). The theory gives the distribution of LC molecules orientation near the structural defect (fig.3).

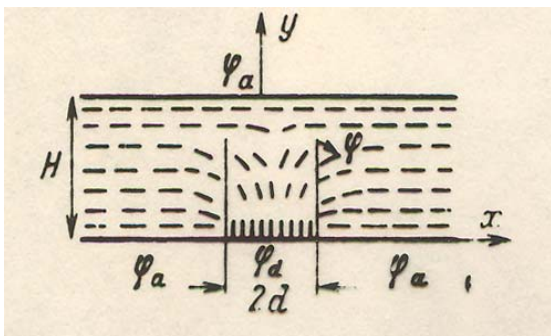

Fig.3. Solitary structural defect and local orientation of LC molecules induced by it 
The NLC molecules orientation induced by structural inhomogeneity is described by equation:

$$
\begin{array}{rrr}
\pi \frac{\varphi(x, y)-\varphi_{a}}{\varphi_{d}-\varphi_{a}}=\operatorname{arctg} \frac{\operatorname{sh}(D-X) \operatorname{ctg} \frac{Y}{2}}{\operatorname{ch}(D-X)+1}+\operatorname{arctg} \frac{\operatorname{Sh}(D+X) \operatorname{ctg} \frac{Y}{2}}{\operatorname{ch}(D-X)+1} ; \\
X=\frac{\pi x}{H}, \quad Y=\frac{\pi y}{H}, & D=\frac{\pi d}{H} .
\end{array}
$$

The theory was used for explanation the results of experimental structural defects examination on the surface of different materials ${ }^{3}$.

The recording process is simple, obvious and informative. One may optimize the image contrast by rotating polarizer and analyzer. The main and virtually the sole operation are to put a uniform NLC layer on the substrate surface. Such layer may be applied using a rotating table or even a simple paintbrush. The heating of NLC up to the temperature of transition to isotropic phase makes the film after cooling more uniform in thickness. The resolution of the NLC layer depends on its thickness and approximately is equal to 2000 lines $/ \mathrm{mm}$. The wetting conditions may be changed by special dopants and surfactants. The reliable results in visualizing the defects on the surface are obtained by repeatable applying and removing NLC layers and observing the stable defects images. The NLC layers are easily removed by a solution of alcohol or acetone. The NLC film distorts the real images of the defects because of its elasticity. To receive the information about the real size of the defects when only the size of their images is known a theory was developed. The theory describes in a simple analytic form the defect's image formation process and its relation to $\mathrm{NLCs}^{2,3}$.

Another problem of LC vision theory is the sensitivity of the NLC technique. It is apparent that such sensitivity depends on the experimental conditions, physical nature of defect origins and the sort of NLC material. On the first stage the phase delay that appears during the radiation transport through the NLC layer was theoretically considered. It was regarded as function of the surface interaction energy fluctuations induced by surface defects. We have to take into account the average interaction energy. In this case the sensitivity $S$ is given by derivative:

$$
S=d \Phi / d E \text {, }
$$

Here $E$ is the fluctuation of interaction energy, $\Phi$ is phase delay. But for final sensitivity determination the methods of information theory of optical image are to be applied. They permit to take into account the thermal LC-noise as well as that of surface itself, the regular surface pattern spectrum and recording device transfer function. This approach gives the limits of NLC technique sensitivity and resolution in visualizing the defects ${ }^{3}$.

Simplicity, efficiency and high sensitivity have given an opportunity of LC vision application in material science, mineralogy, crystallography, thin film technology, medicine and biology. The operation with OPM and NLCs as recording media is very simple process and not need the high quality experts for making the experiments. The students receive necessary practice after few days of study. It takes a short time to make experiments but a long time to understand the results. The main results in student researches are published in papers ${ }^{4-9}$.

\section{THE MAIN RESULTS IN STUDENTS' RESEARCHES BASED ON NEW OPM APPLICATION}

For students' researches illustration we select the investigations devoted to the most attractive results in the field of biophotonics: grippe virus, cancer and erythrocyte pathology detecting.

\subsection{Grippe viruses detecting}

The problem of grippe viruses detecting is important because of its possible dangerous epidemic and pandemic scale all around the world. There are some methods of grippe viruses detecting based on application of dyes and fluorescence technique ${ }^{10}$. Unfortunately all these methods are impossible to visualize directly the grippe virus modifications. They also need few days for detecting and consists many steps of operation. In order to avoid the disadvantages we suggested to apply new contact technique based on liquid crystal (LC) application: LC vision ${ }^{3}$. 
The first effective results of LC application in viruses detecting were described in patent ${ }^{11}$. Its main particularity was in usage of polyaniline (PA) layer to obtain a good adhesion of antibody material to the glass plate. Another technique based on deficit lyotropic LC application was described in paper ${ }^{12}$. The technique is expensive and also impossible for direct visualization the grippe viruses modification.

In our paper we suggest a simple, expressive and informative method based on cheap nematic LC application that didn't need the usage of PA layer. On glass plate the antibody-viruses sandwich was placed and coated with thin LC layer of mixture MBBA:EBBA. In the case of antibody-viruses unconformity the complimentary reaction doesn't take place that may be directly observed through polarizing microscope (fig.4). In the case of antibody-viruses conformity the complimentary reaction takes place that visualize quite different surface structure with crystal-like segments (fig.5). The student Michailova-Kad'ikova T.S took part in the experiments.

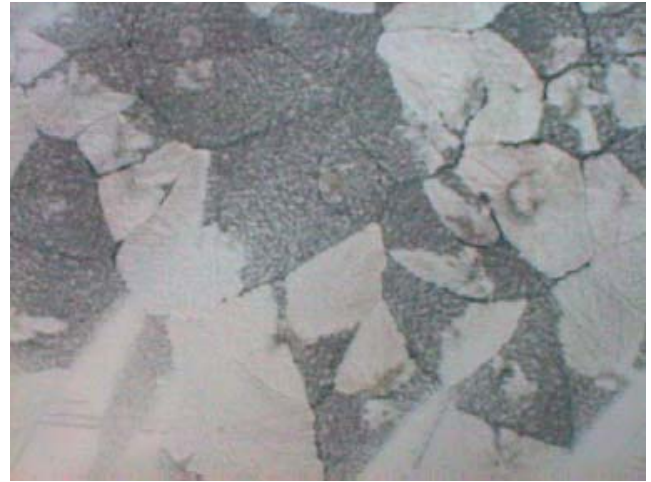

Fig.4. The structure of the boundary antibodyviruses-LC layer in the case of their uncomplimentary reaction. $100^{\times}$

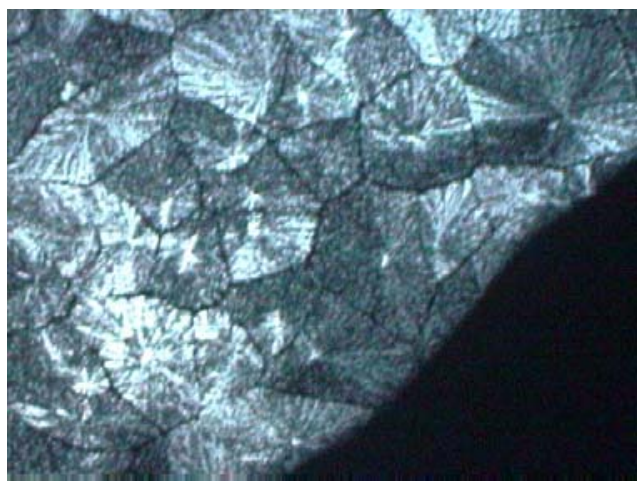

Fig.5. The crystal-like structure of the boundary antibody-viruses-LC layer in the case of their complimentary reaction. $100^{\times}$

Summarizing the results of our investigations it is possible to conclude that new simple and expressive technique based on NLC application to grippe viruses is developed ${ }^{8}$. It promises the possibility for direct observation of the surface structures in the case of viruses mutation.

\subsection{Malignant tumors detecting}

The problem of malignant tumors is one of the most actual in modern medicine. It consists not only of the development of effective patients' treatment methods but in objectification of microscopic neoplasm diagnosis. The routine diagnostic methods are based on optical microscopy modifications in view of spectral, luminescent and fluorescent features of the growth images. The essence of luminescent and fluorescent methods is based on the properties of some photosensitive materials to be collected in malignant tissues in the much greater degree, than the surrounded normal tissues, that under certain conditions allows receiving luminescent and fluorescent images of tumors. These methods are suitable for detecting malignant growth on outside surfaces of a body in dermatology and cosmetology, and also on internal surfaces observed with the application of endoscope's technique. Advantage of the methods is an opportunity to study the efficiency of undertaken medicine measures in real time. However the methods are unacceptable for microscopic diagnosis.

For traditional histological diagnosis tissue sections of 4-5 $\mu$ thick are prepared of the block after freezing or embedding in paraffin. Then sections are placed on an object glass and treated with organic dyes. Haematoxylin and eosin are usually used as dyes and that impart different colors to the nucleus and cytoplasm in the cell. The malignant character of a tumor is verified by observing in light microscope cell atypism features and invasive particularities of malignant growths. The disadvantage of the method is, that the observable distinctions in some cases are too week, that the diagnosis is made in the greater degree on 
intuition of the experts, rather than on objective criterion revealing. The absence of objective criteria for diagnosis can sometimes result in irreparable mistakes.

The new optical criterion for objective diagnosis of malignant growth in tissues of human beings and animals based on NLC technique is suggested and confirmed with experiments ${ }^{13,14}$.

The first experiments with NLCs on detection of the pathological changes in sections of human epithelial tissues have given the positive results (Fig.6). The experiments discovered different concentration of keratin in ripe and "young" cells that was distinguished by different orientation of NLC molecules.

A

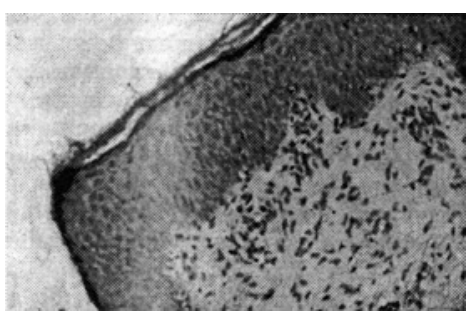

B

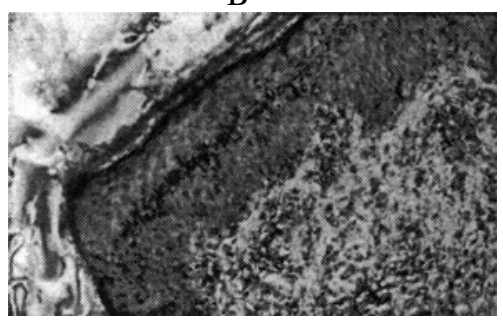

Fig.6. The sections of epithelial tissues observed through light microscope (A) and with NLC technique (B), visualized the different concentration of keratin in cells.

That was the reason why the regular comparative investigations of malignant and benign tumors were carried out (Fig.7).

A
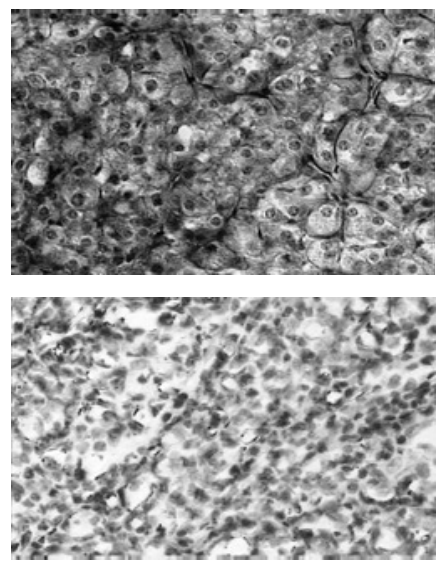

B
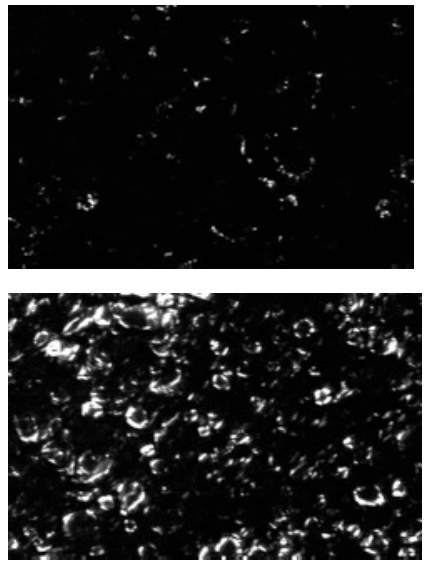

Fig.7. LC vision in detecting rental cancer (upper rank) and stomach cancer (low rank). A - tissues decorated with dyes. B - tissues decorated with NLC.

Only frozen tissue sections were suitable for experiments. Routine histology was used for identification of malignant areas in micro preparates. In order to avoid the NLC molecules orientation induced by plotting operation the mixture of NLC and the solvent were used. The solvent transferred NLC to isotropic phase. After the mixture was plotted on the tissue surface the solvent evaporated and NLC orientation was obtained only by structural topography.

Through a light microscope with crossed analyzer and polarizer in all investigated cases malignant growth decorated with NLC looked black (except for clear distinct vessels and elements of linkage skeleton). At the same time NLC molecules on normal tissues and benign tumors always looked white or color. The similar pictures were observed in dog and human tissues. 
The essentially new result was the proof of an opportunity of LC vision use for diagnostics of tissues in vivo (Fig.8). The revealed phenomenon was suggested as a new objective diagnostic criterion for malignant growth. To confirm the reliability of the offered criterion it was necessary to realize careful analysis and many of experiments.

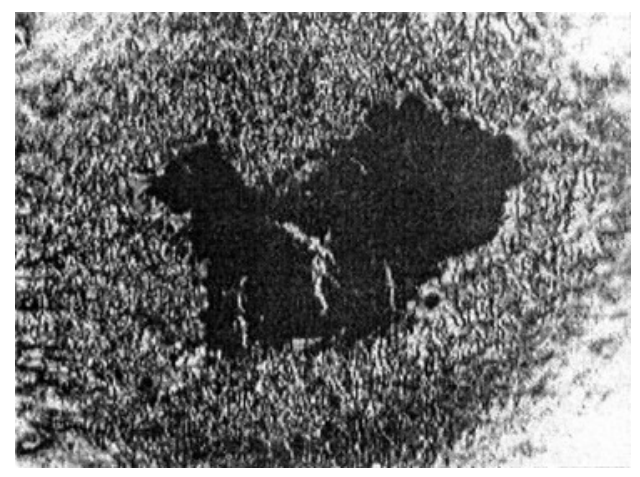

Fig.8. Malignant smear of a mouse tissue decorated with MBBA (dark area). Magnification $25^{x}$.

It is known, that the orientation conditions of NLC molecules on a solid surface depends on relation between the surface tensions on the boundary of the materials. When the surface tension of liquid crystal YLC is bigger than the surface tension of a substrate $Y_{S}$, the NLC molecules exhibit homeotropic alignment. If $Y_{L C}<$ $Y_{s}$, the NLC molecules alignment is tilted. When viewed through a microscope in crossed analyzer and polarizer the areas with homeotropic alignment of molecules look black (no light passes through the analyzer). If the molecules have orientation close to planar, such areas look white (intensity of light in connection with the arisen phase delay is distinct from zero) (Fig.9a, b).

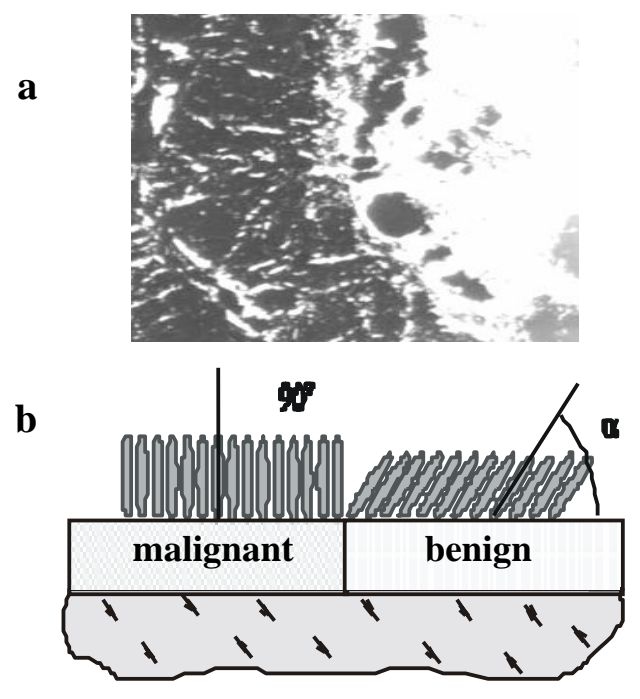

Fig.9. Photo of rectal cancer (adenocarcinoma): malignant (left) and benign (right) tissues decorated by NLC layer (a). The model of NLC molecules orientation on tissues (b).

All this implies that NLC layer is a unique recording medium for visualizing the distribution of surface tension over the investigated surface. As structural defects cause the local change of surface tension the NLC technique gives the unique possibility to observe directly the regions of the surface with local defects. The specified property allowed us to formulate a new biophysical criterion for objective diagnosis of malignant 
tumors. Thus the orientation of NLC molecules on the substrate is the micro molecular characteristic of the surface determined energy of NLC molecules coupling with the substrate. The different NLC molecules orientation on investigated tissues means a difference in anchoring energy of NLC molecules on a surface of malignant and not malignant tumors. For confirmation the reliability of entered criterion it was necessary to confirm the distinction of surface characteristics by independent experiments on macromolecular level, for example in direct measurement of malignant and not malignant tissues surface tension.

It is known, that on a number of biophysical and biochemical characteristics the malignant tumors differ from normal tissues. However in the scientific literature there is no information on their surface tension value.

The research of tissue surface tension was made by wetting contact angles definition of investigated surfaces on the boundary with reference liquids. The photographic method allowing to a drop's profile at counter illumination was used for receiving its shadow image. The samples were fixed in $10 \%$ formalin solution, of which on freezing microtome 5-7 $\mu$ thick sections were prepared. Some sections contained boundary areas of malignant and not malignant tissues, which could be observed simultaneously. Till two consecutive sections from each sample were used. One of them was exposed to traditional dyes processing for revealing malignant areas. Another section was located on subject glass, and after drying on it one drop (or several drops) 1-2 $\mathrm{mm}$ in diameter of a reference liquid was plotted. The sample was located on a table, which could smoothly move in vertical and two perpendicular horizontal directions. The drop was shined with a collimated beam for recording with camera. For absorption of an infrared range of radiation the light source equipped with special filter. Water and MBBA were used as reference liquids.

The profile of a drop with current of time changed, as the researched tissues absorbed some part of a liquid. There for the recording of a drops profile was carried out repeatedly through the certain intervals of time after their drawing (through 1/2, 2, 5 and 10 minutes). The reflective mirror was used for tangent construction to the drop profile up to complete sphere, which allowed measuring contact angles with the increased accuracy (Fig.10).

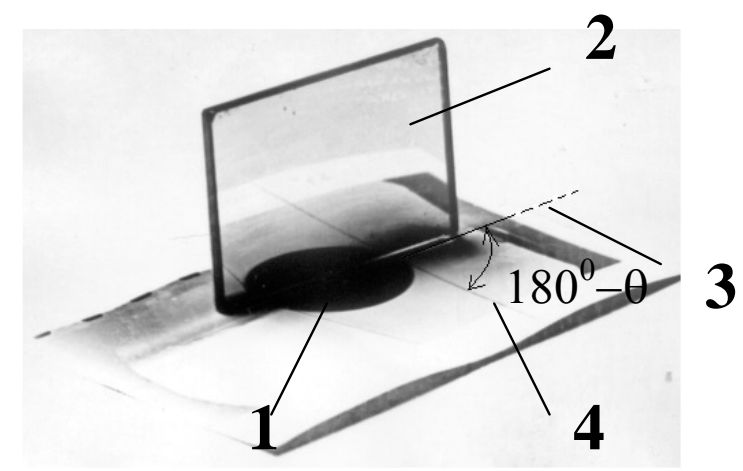

Fig.10. Mirror technique for tangent construction and contact angle $\theta$ measurement: 1-droplet's profile; 2reflective mirror; 3-horizontal line; 4- tangent line.

The results of contact angles measurements of reference liquids on the surface of various types of malignant tumors, through the fixed intervals of times after drawing droplet are given in Table 1.

The comparative experiments carried out on 10 samples of tissues have shown, that in all cases the wetting contact angles of reference liquids on healthy tissues were more, than on malignant tumors. The difference makes essential size reaching on the average 30\% from absolute value of measuring parameter.

For the surface tension $\gamma_{s v}$ calculations of malignant and normal tissues Young and Newman equations were used:

$$
Y_{S L}=2\left(Y_{L V} \times Y_{S V}\right)^{1 / 2} e^{-0,00015}\left(Y_{L V}-Y_{s V}\right)^{2}
$$

$s$ - index of solid surface; $L$ - liquid; $v$ - vapor 


$$
\mathrm{Y}_{S L}=\mathrm{Y}_{S V}-\mathrm{Y}_{L V} \operatorname{Cos} \theta
$$

From our experiments $Y_{L}$ for MBBA at room temperature is $30 \mathrm{dyn} / \mathrm{cm}^{2}$. The results of calculations are: $Y_{s v}=26 \mathrm{dyn} / \mathrm{cm}^{2}$ for malignant tissues; $Y_{s v}=32 \mathrm{dyn} / \mathrm{cm}^{2}$ for healthy tissues. The results of calculations and experiments are in good agreement with F. Kahn rule: $\gamma$ of malignant tissue (homeotropic orientation) $<y$ of MBBA < $y$ of normal tissue (planar orientation) ${ }^{15}$.

Table 1. Contact angles of reference liquids on surfaces of various tissues

\begin{tabular}{|c|l|c|c|c|c|c|c|c|c|}
\hline \multicolumn{1}{|c|}{ LIQUID SAMPLE } & \multicolumn{3}{|c|}{$\mathrm{H}_{2} \mathrm{O}$} & \multicolumn{3}{c|}{ MBBA } \\
\hline \multicolumn{1}{|c|}{ Minutes } & 0,5 & 2 & 5 & 10 & 0,5 & 2 & 5 & 10 \\
\hline 1 & $\begin{array}{l}\text { Abdominal desmoid (fibrous tissue } \\
\text { malignant tumor of the abdominal wall) }\end{array}$ & 79 & 74 & 64 & 58 & 36 & 35 & 30 & 30 \\
\hline 2 & $\begin{array}{l}\text { Breast fibroadenoma (fibrous tissue } \\
\text { benign tumor) }\end{array}$ & 84 & 74 & - & 74 & 30 & 25 & 20 & 18 \\
\hline 3 & $\begin{array}{l}\text { Breast fibrosarcoma (fibrous tissue } \\
\text { malignant tumor }\end{array}$ & 71 & 70 & 65 & 58 & 40 & 40 & 35 & 35 \\
\hline 4 & $\begin{array}{l}\text { Scar of the esophagus (overgrowth of } \\
\text { normal fibrous tissue) }\end{array}$ & 83 & 76 & 74 & 65 & 15 & 12 & 10 & 10 \\
\hline 5 & $\begin{array}{l}\text { Sclerotic ovary (overgrowth of normal } \\
\text { fibrous tissue) }\end{array}$ & 90 & 84 & 81 & - & 30 & 25 & 22 & 20 \\
\hline 6 & Scirrhous breast cancer & 60 & 48 & 35 & 27 & - & - & - & - \\
\hline 7 & $\begin{array}{l}\text { Uterine leyomyoma (smooth muscle } \\
\text { tumor) }\end{array}$ & 68 & 62 & 55 & 49 & 20 & 20 & 15 & 15 \\
\hline 8 & $\begin{array}{l}\text { Skin melanoblastoma of the dog } \\
\text { (malignant melanocytic tumor) }\end{array}$ & 73 & 61 & 51 & 52 & 40 & 40 & 40 & 40 \\
\hline 9 & $\begin{array}{l}\text { Human skin melanoblastoma } \\
\text { (malignant melanocytic tumor) }\end{array}$ & 48 & 48 & 37 & - & 40 & 40 & 35 & 35 \\
\hline 10 & \begin{tabular}{l} 
Normal dog skin fibrous tissue \\
\hline
\end{tabular} & 86 & 78 & 67 & 45 & 35 & 35 & 30 & 30 \\
\hline
\end{tabular}

To conclude the direct determination of malignant and not malignant surface tension values explain the different orientation of NLC molecules on their surfaces.

In our paper the new model for explanation the difference in surface tension value on the malignant and benign tumors is discussed. The model is illustrated by the figure below (Fig. 7).

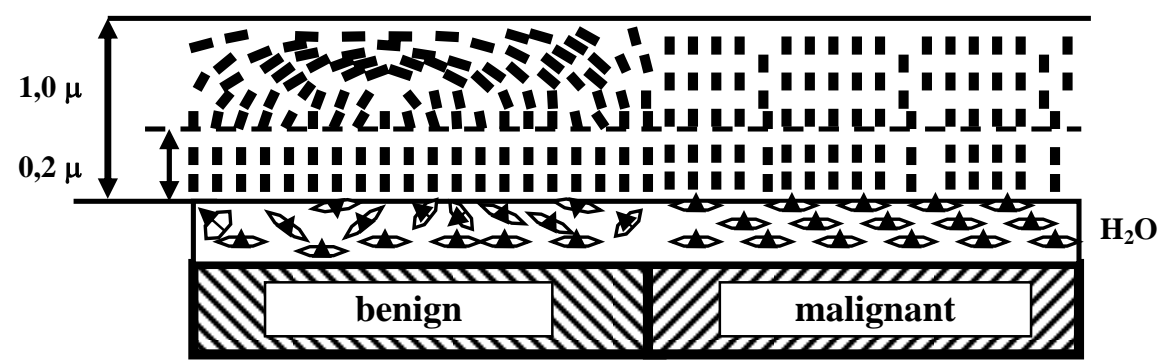

Fig.11. The water on the surface of malignant tissues has much higher order parameter in comparison with the water on benign tissue 
Many scientists observed the interface layer of structural water on the surface of tissues. The explanation of the difference in surface tension values is based on the particularities of the different parts of the interface layer. It is known that the water on the surface of malignant tissues has much higher order parameter in comparison with the water on benign tissue ${ }^{16}$. The difference is explained by higher concentration of the protein and less concentration of lipids in the water on malignant tissues. It means that the anchoring energy of NLC on the surface of such water must be higher in comparison with anchoring energy on the surface of the water on benign tissue. In the first case the NLC molecules have the homeotropic orientation in the whole thickness of NLC layer while in the second case the surface Fredericks transition is observed ${ }^{17}$.

The application of LC vision in medicine opens new horizons in cancer nature investigations. The first experiments demonstrated that LC vision seems to be useful for leukemia express detecting. The student Efimova T.A. took part in the experiments.

\subsection{Erythrocytes pathology detecting}

It was suggested to use OPM for detecting the erythrocytes behavior in NLC matrix. The smear of human blood was dried up at the open air without pollution admittance. The thin layer of NLC was applied on the blood smear and observed in OPM. The results of NLC structure investigation are presented at fig.12. It was demonstrated that healthy erythrocytes in NLC matrix having previously oval form change their configuration to rectangle form. The reason of form change is explained by forces of intermolecular interactions in system. Erythrocytes with pathology were deformed because of their elasticity violation.

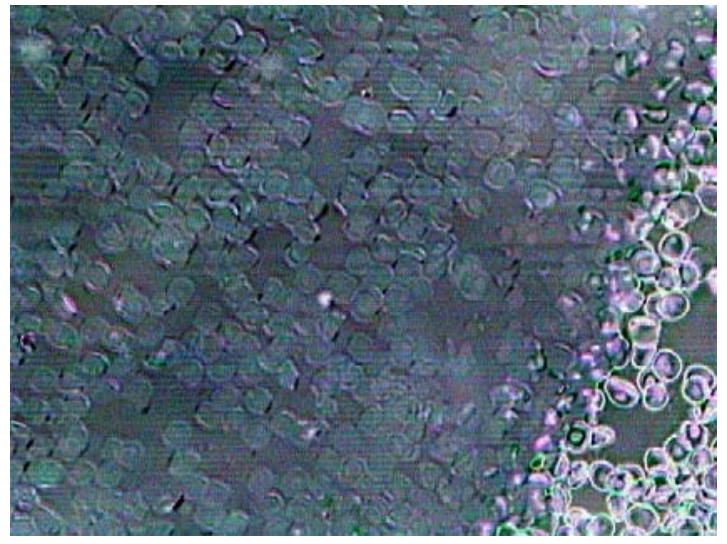

Fig.12. Blood smear image after applying NLC thin layer. The boundary divides erythrocytes with (left) and without NLC (right). The normal erythrocytes formed the rectangle figures while erythrocytes with pathology formed deformed rectangle figures. $5 \mathrm{CB}, \mathrm{t}=20^{\circ} \mathrm{C}, 250 \mathrm{x}$.

The results were confirmed by independent laser diffraction experiments. LC vision is a new independent technique for erythrocytes pathology detecting. The post-doc Smirnova I.G. took part in the experiments.

Summarizing the results of our investigations it is possible to conclude that new simple and expressive technique based on OPM with NLCs application testify to wide possibilities in examination the surface properties of biological objects.

We can recommend the studies of new objects and materials surfaces with new optical microscope for education and training in optics and photonics. 


\section{References}

1. Tomilin M.G., Pestov S.M. The properties of liquid crystal materials. St.-Petersburg, Polytechnika (in Russian). 2005. 296 p.

2. Tomilin M.G. New physical method to study characteristics of the surface coated by NLCs // Mol. Cryst. Liq. Cryst. 1990. Vol.193. P.7-11.

3. Tomilin M.G. The interaction of liquid crystals with the surface. St.-Petersburg, Polytechnika (in Russian). 2001. 325 p.

4. Ivanov V.F., Tomilin M.G., Yablokov M., Gribkova O., Isakova A., Vannicov A., Ivanova V., Rakutina R. Induced optical activity of polyaniline interpolymer complexes and their use in virology and medicine // European polymer congress 2005, Moscow, Russia, P2.2-7, Ref 4119.

5. Tomilin M.G., Povzun S.A., Gribanova E.V., Efimova T.A. New criterion of cancer detection based on LC molecules orientation // Mol. Cryst. Liq. Cryst., 2001, V. 367, p. 133-141.

6. Kerpeleva S.Yu., Terukov E.I., Tomilin M.G. LC vision: new application to silicon surface defects testing // Mol. Cryst. Liq. Cryst., 2001, V. 368, p. 1-8.

7. Tomilin M.G., Kuznetzov P.A., Galyametdinov Yu.G. LC vision: the application to magnetic material domains investigations // Book of abstracts: $20^{\text {th }}$ ILCC, July 4-9, 2004. Ljubljana, Slovenia, SURFPO13, p. 745.

8. Tomilin M.G., Michailova-Kad'ikova T.S., Stepanova A.A. LC vision: new application in grippe viruses detecting // Abstracts of ICOL-2005, IRDE, Dehradun, India, pP-BP-1, p. 24.

9. Bessmeltsev S.S., Smirnova I.G., Stafeev S.K. Tarlykov V.A., Tomilin M.G. LC Vision application to erythrocytes pathology detecting // Book of Abstract ECLC2007, 9-th European conference on Liquid Crystals, Lisbon, Portugal, July 2-6, 2007.

10. Song J., Cheng Q., Zhu S., Stevens R.C. "Smart" materials for biosensing devices: cell-mimicking supramolecular assemblies and colorimetric detection of pathogenic agents // Biomedical Microdevices: 4:3. 2002. P. 213-221.

11. Ivanov V.F., Ivanova V.T., Tomilin M.G., et al. Method of viruses detecting. Patent application № 2004135136/13(038228), 02.12.2004.

12. Shiyanovskii S.V., Schneider T., Smalyukh I.I. et al. Real-time detection based on director distortion around growing immune complexes in lyotropic chromonic liquid crystals // Phys. Rev. E 71. 2005. 020702-1-020702-4.

13. Tomilin M.G., Povzun S.A. New criterion of cancer detection based on NLC molecules orientation // Abstracts of 18 ILCC, Sendai, Japan, 24D-56-P, p. 173, 2000.

14. Tomilin M.G., Povzun S.A., Kurmashov A.F. LC Vision: the application to the cancer nature investigations // Book of Abstracts, ECLC2003, Jaca, Spain, O25, 2003.

15. Kahn F.J., Taylor C.N., Shonhorn H. Surface-produced alignment of LCs // Proceed. IEEE, 1973. Vol.61, P.823-825.

16. Downing J.E. "Nuclear water content during carcinogenesis", Cancer, 1962. Vol.15, № 6. P.11761180 ,

17. Blinov L.M., Sonin A.A. "The interaction of NLCs with anisotropic substrates", Mol Cryst. Liq. Cryst., 1990. Vol.179. P.13-25. 